\title{
Rowing Over the Edge: Nonfunctional Overreaching and Overtraining Syndrome as Maladjustment- Diagnosis and Treatment From a Psychological Perspective
}

\author{
Daniel Birrer \\ Swiss Federal Institute of Sport Magglingen
}

\begin{abstract}
A rigorous training schedule with insufficient recovery can lead to nonfunctional overreaching (NFOR) or overtraining syndrome (OTS). Research has suggested the multifactorial etiology of these phenomena. Stressors that contribute to and are symptoms and consequences of NFOR and OTS and adjustment disorder are almost identical. In this case study of an elite rower, the author illustrates an intervention approach that can be taken when overtraining is viewed as a sport-specific form of adjustment disorder. The intervention involved treatment that improved the athlete's awareness of his basic biopsychosocial processes, developed sources of self-worth beyond athletic performance, and challenged his 1-dimensional athletic identity. The intervention included cognitive-behavioral therapy methods (e.g., autogenic training) and mindfulness- and acceptancebased interventions to enhance the athlete's psychological flexibility. Mood monitoring was used as a diagnostic and evaluative instrument. Intervention effectiveness was evaluated through an in-depth interview with the athlete. The consulting sport psychologist also engaged in reflection about treatment effectiveness and predominant challenges. Challenging the athlete and clarifying his personal values were judged to be very important. Evaluation suggested that viewing NFOR and OTS as forms of adjustment disorder may help us recognize the multifaceted nature of an athlete's maladjusted state and widen treatment options.
\end{abstract}

Keywords: adjustment disorder, psychological flexibility, staleness, underperformance

Training imposes stress on athletes, exhausting their biopsychosocial system to trigger an adaptation process, allowing them to cope better with the same stressor in the future (Meeusen et al., 2013). However, "some sports, like rowing, are about being mentally tough, and they're about mastering the pain and pushing your body hard. That kind of sport culture encourages overtraining" (Richardson, Andersen, \& Morris, 2008, p. 159). The reported prevalence of overtraining varies widely, ranging from $5 \%$ to $60 \%$ (Birrer, Lienhard, Williams, Röthlin, \& Morgan, 2013; Kreher, 2016). Given the severity of its symptoms and its impairment to quality of life, overtraining phenomena must be considered serious and career-threatening events. Using the story of an elite rower, I illustrate the advantages of diagnosing and treating nonfunctional overreaching (NFOR) and overtraining syndrome (OTS) by viewing them as forms of maladjustment (Jones \& Tenenbaum, 2009).

\footnotetext{
(C) 2019 The Authors. Published by Human Kinetics, Inc. This is an Open Access article distributed under the terms of the Creative Commons Attribution-NonCommercial 4.0 International License, CC BY-NC 4.0, which permits the copy and redistribution in any medium or format, provided it is not used for commercial purposes, no modifications are made, and appropriate credit is given. See http:// creativecommons.org/licenses/by-nc/4.0. This license does not cover any third-party material that may appear with permission in the article. For commercial use, permission should be requested from Human Kinetics, Inc., through the Copyright Clearance Center (http://www.copyright.com).
}

The author is with the Elite Sport Dept., Swiss Federal Inst. of Sport Magglingen (SFISM), Magglingen, Switzerland. Address author correspondence to daniel. birrer@baspo.admin.ch.

\section{Overtraining and Maladjustment: Theoretical Considerations}

Athletes train to enhance and optimize their performance. Studies investigating the influence of training volume, intensity, and frequency on athletic performance have generally found that athletes improved their performance with increases in training load (Gabbett, 2016). However, a rigorous training schedule with insufficient recovery, probably also caused by sources of nontraining stress, may lead to maladaptive responses in the form of injury and performance decrements. Unexplained performance decrements despite adequate rest are discussed in the literature using terms such as overtraining, overreaching, staleness, or underrecovery (Kreher, 2016). In awareness that the variety of terms used to describe this phenomenon could cause confusion, I define the relevant terms before presenting the case of the rower.

\section{NFOR and OTS: Definition, Signs, and Genesis}

In a European College of Sport Science (ECSS) and the American College of Sports Medicine (ACSM) joint consensus statement on OTS, Meeusen et al. (2013) emphasized the use of the term syndrome to express its multifactorial etiology. Regardless of the terminology used, training is not generally regarded as the sole causative factor. Other factors - such as inadequate nutrition, illness, and psychosocial stressors-also lead to prolonged maladaptation with prolonged and inexplicable underperformance. In the ECSS/ ACSM definition, on which this paper is based, overtraining has been viewed as a process of intensified training that can result in functional overreaching, NFOR, or OTS (see Figure 1). 


\begin{tabular}{|c|c|c|c|c|}
\hline Process & $\begin{array}{l}\text { Training } \\
\text { (overload) } \\
\text { Stress-recovery } \\
\text { balance }\end{array}$ & \multicolumn{3}{|c|}{ Training increase / Life stress increase } \\
\hline Outcome & Acute fatigue & $\begin{array}{l}\text { Functional } \\
\text { OR }\end{array}$ & $\begin{array}{l}\text { Non-functional } \\
\text { OR }\end{array}$ & $\begin{array}{l}\text { Overtraining } \\
\text { syndrome }\end{array}$ \\
\hline Mood change & $\begin{array}{l}\text { None or } \\
\text { 'Good mood' } \rightarrow \\
\text { 'Vigour' } \rightarrow\end{array}$ & $\begin{array}{l}\text { 'Good mood' ע } \\
\text { 'Vigour' ע } \\
\text { 'Fatigue' } \lambda\end{array}$ & $\begin{array}{l}\text { 'Good mood'У } \\
\text { 'Vigour'У } \\
\text { 'Fatigue' } \text { 'Depressed mood' } \\
\text { 'Ded }\end{array}$ & $\begin{array}{l}\text { Same as } \\
\text { NFOR } \\
\text { + possibly } \\
\text { Anxiety } \\
\text { Depression }\end{array}$ \\
\hline $\begin{array}{l}\text { Time } \\
\text { dimension }\end{array}$ & Short-term & $\begin{array}{l}1 \text { day - } \\
2 \text { weeks }\end{array}$ & $\begin{array}{l}2 \text { weeks - } \\
2 \text { months }\end{array}$ & 2 months \\
\hline
\end{tabular}

Figure 1 - Possible presentation of the different stages of training, functional overreaching (OR), nonfunctional overreaching, and overtraining syndrome according to Meeusen et al. (2013), with symptomatic mood changes as stage indicators.

Functional overreaching is a desired outcome of a planned training process in which an athlete suffers only temporary performance impairment. With appropriate rest and recovery, ranging from several days to up to 2 weeks, performance can be improved through supercompensation despite overreaching. This process is commonly referred to as tapering (Bosquet, Montpetit, Arvisais, \& Mujika, 2007). However, sometimes, an athlete is not able to adapt positively as expected, given the athlete's experience during the previous periodized training process. If, after 14 days of total rest or training reduction of at least $20 \%$, an athlete cannot return to the expected performance level, then NFOR might be diagnosed. In addition to the cardinal symptoms of high fatigue and reduced performance, Meeusen et al. (2013) cited other symptoms including psychological distress, psychological disturbance, and physiological consequences (e.g., higher prevalence of infections, most commonly those of the upper respiratory tract; see Table 1).

It is difficult to distinguish between NFOR and OTS. The signs and symptoms of each are often the same, but OTS typically presents with abnormal physiology, more severe symptoms, and a longer duration of decreased performance (more than 2 months). Furthermore, the diagnoses of NFOR and OTS are complex due to the retrospective nature of their definitions. Adequate training reduction and rest of at least 14 days in duration are crucial. In addition, it is vital to exclude organic diseases, infections, and lifestyle factors-such as dietary caloric restriction (negative energy balance), insufficient carbohydrate and/or protein intake, and iron deficiency - and to identify initiating events or triggers (Meeusen et al., 2013). Because overtraining is a phenomenon of excessive sporting behavior, the most certain trigger is a trainingrecovery miscalculation together with other sport-specific causes (see Table 2). However, as shown in Table 2, the apparent multifactorial etiology of NFOR and OTS means that a combination of multiple life stressors and a complex set of psychological factors are likely responsible for the prolonged maladaptation (Armstrong \& VanHeest, 2002; Meeusen et al., 2013; Richardson et al., 2008).

To understand the mechanisms involved in the evolution of NFOR and OTS, it is also important to understand an athlete's sociocultural context, which can be viewed in terms of both risk and protective factors that influence stress load and recovery (Richardson et al., 2008). Risk factors can also be seen as stressors. Important factors stem from sport culture and societal norms, influences, and expectations. These include situational factors, as well as interpersonal influences from coaches, parents, and significant others, which produce patterns of reinforcement for overtraining and recovery behavior (see Table 2). Additional intrapersonal variables may include fitness level, stage of physical development, seeking love and approval for performance, and personality characteristics such as fear of failure, perfectionism, performance-based self-esteem, and athletic identity (Richardson et al., 2008).

Athletes with early signs of NFOR and OTS typically exhibit an increase in total mood disturbance that follows a specific pattern (see Figure 1). Specifically, during phases of peak overload, positive mood decreases and fatigue increases. Athletes with coping inadequacy will also react with decreased vigor. A maladaptive behavioral response is further characterized by ignorance or denial of the signs of a stress-recovery imbalance or an inability to react adequately to the stress situation. Therefore, athletes who find themselves underperforming may be tempted to compensate for their poor performance by training more often and more intensely. As a result of unsuccessful attempts to regain their desired performance level through an increase in training volume and intensity, good mood will continue to decrease and fatigue will continue to increase. In addition, vigor will decrease and depressed mood will increase (see Figure 1). A less severe outcome will be NFOR and a more severe outcome will be OTS. Generally, OTS has been characterized by a significant increase in depressed mood, which might be similar to that observed in clinical depression. In one study, $80 \%$ of OTSdiagnosed athletes showed signs of clinical depression (Morgan, Brown, Raglin, O'Connor, \& Ellickson, 1987). A stable and moderate to high increase in depressed mood can be regarded as a cardinal sign of maladjustment of the biopsychosocial system, resulting in NFOR or OTS (Meeusen et al., 2013; Schwenk, 2000).

\section{Adjustment Disorder: Definition, Signs, and Genesis}

Adjustment disorder is a common problem, with a reported prevalence of $1-2 \%$ in the general population (Zelviene \& Kazlauskas, 2018). It is present when stressful expected or unexpected events cause an individual to be confused and lost and negatively affect the individual's behavior, well-being, and mental health (American Psychiatric Association, 2013). Typically, one or more events occur to derail someone who is subsequently unable to adapt as well as the average person in the same situation. The symptoms are 
Table 1 Symptoms and Consequences of Adjustment Disorder Found in the Literature and Their Occurrence as Symptoms and Consequences of NFOR or OTS

\begin{tabular}{|c|c|c|}
\hline Symptoms and consequences & $\begin{array}{l}\text { Occurrence in NFOR } \\
\text { or OTS }\end{array}$ & $\begin{array}{l}\text { Comments regarding occurrence in NFO and OTS } \\
\text { literature }\end{array}$ \\
\hline \multicolumn{3}{|l|}{ Symptoms } \\
\hline sadness/depressed mood & $\checkmark$ & \multirow{8}{*}{$\begin{array}{l}\text { Symptoms of NFOR and OTS are described as symptoms of } \\
\text { prolonged training distress, such as performance decrements, psy- } \\
\text { chological disturbance, and psychological distress, especially mood } \\
\text { disturbances such as decreased vigor, increased fatigue, depression, } \\
\text { loss of motivation, drowsiness, apathy, devaluation or cynicism } \\
\text { toward the sport, irritability, agitation, restlessness, emotional and } \\
\text { physical exhaustion, lack of mental concentration, memory pro- } \\
\text { blems, anxiety, awakening unrefreshed, and heavy, sore, and/or stiff } \\
\text { muscles (Armstrong \& VanHeest, 2002; Jones \& Tenenbaum, 2009; } \\
\text { Kreher, 2016; Meeusen et al., 2013; Richardson et al., 2008). }\end{array}$} \\
\hline hopelessness & $\checkmark$ & \\
\hline decreased motivation & $\checkmark$ & \\
\hline low mood & $\checkmark$ & \\
\hline loss of pleasure & $\checkmark$ & \\
\hline exhaustion & $\checkmark$ & \\
\hline anxiety/excessive worry/fear & $\checkmark$ & \\
\hline nervousness & $\checkmark$ & \\
\hline restlessness & $\checkmark$ & \\
\hline poor concentration & $\checkmark$ & \\
\hline irritability & $\checkmark$ & \\
\hline \multicolumn{3}{|l|}{ decreased self-esteem } \\
\hline anger or disruptive behavior & \multirow[t]{3}{*}{$\checkmark$} & \\
\hline \multirow{2}{*}{\multicolumn{2}{|c|}{$\begin{array}{l}\text { loneliness/feeling isolated } \\
\text { suicidal ideation }\end{array}$}} & \\
\hline & & \\
\hline $\begin{array}{l}\text { sense of being overwhelmed/feeling as if } \\
\text { trapped and have no other options }\end{array}$ & $\checkmark$ & \\
\hline \multicolumn{3}{|l|}{ Consequences } \\
\hline $\begin{array}{l}\text { significant impairment in personal, } \\
\text { family, social, occupational, or other } \\
\text { important areas of functioning such as ... }\end{array}$ & $\checkmark$ & \multirow{6}{*}{$\begin{array}{l}\text { The cardinal consequence or symptom in NFOR or OTS is persistent } \\
\text { underperformance despite adequate rest or significant training } \\
\text { reduction of } 14 \text { days. In addition, insomnia, weight loss, anorexia, } \\
\text { increased immunological suppression, illness (most commonly } \\
\text { upper respiratory tract infections), decreased heart rate at all exercise } \\
\text { intensities (including at exhaustion), increased rating of perceived } \\
\text { exertion at submaximal intensities, reduced blood lactate concen- } \\
\text { tration at both maximal and submaximal intensities, and altered } \\
\text { cognitive performance above the lactate threshold are described } \\
\text { (Armstrong \& VanHeest, 2002; Jones \& Tenenbaum, 2009; Kreher, } \\
\text { 2016; Meeusen et al., 2013; Richardson et al., 2008). }\end{array}$} \\
\hline decreased performance in work or school & $\checkmark$ & \\
\hline avoiding social interactions & & \\
\hline $\begin{array}{l}\text { insomnia/poor sleep/change in sleeping } \\
\text { habits (too much or too little) }\end{array}$ & $\checkmark$ & \\
\hline lack of or increased appetite & & \\
\hline frequent crying & $\checkmark$ & \\
\hline
\end{tabular}

Note. NFOR = nonfunctional overreaching; OTS = overtraining syndrome. Symptoms and consequences of adjustment disorder based on DSM-5 and ICD-10. The characteristic feature is often a mixture of emotional and behavioral symptoms. Internalizing or externalizing symptoms often coexist, although one or the other may predominate.

not of sufficient specificity or severity to justify the diagnosis of another mental or behavioral disorder and must not be part of normal bereavement. When the stressor is removed or the individual has begun to adjust and cope, symptoms typically subside within 3-6 months.

The genesis and course of adjustment disorder are quite personal. Table 1 gives an overview of the most relevant symptoms. However, unambiguous symptomatological criteria in the Diagnostic and Statistical Manual of Mental Disorders, 5th edition (DSM-5; American Psychiatric Association, 2013) and the International Statistical Classification of Diseases and Related Health Problems, 10th revision (ICD-10; World Health Organization, 2016) are lacking (Casey \& Bailey, 2011). Nonetheless, symptoms of low mood, sadness, worry, anxiety, insomnia, and poor concentration in response to a recent stressful event are likely indicators of adjustment disorder. Because major depression can present similarly, clinical judgment is of great importance in the diagnosis of adjustment disorder. Typically in adjustment disorder, mood disturbance is more noticeable when the person is cognitively engaged with the predisposing event—such as when he or she is speaking about it - whereas at other times, the individual's mood is normal (Casey \& Bailey, 2011).

The ICD-11 definition of adjustment disorder offers a slightly new perspective (World Health Organization, 2018). The key symptomatology is still regarded as a maladaptive reaction to an identifiable psychosocial stressor or multiple stressors (e.g., divorce or conflicts at work), but the disorder is characterized by a preoccupation with the stressor or its consequences, including excessive worry, recurrent, and distressing thoughts about it and/or constant rumination about its implications, as well as by a failure to adapt to the stressor. For a diagnosis to be made, it must cause significant impairment in important areas of functioning. Depression, anxiety, avoidance, and impulsivity are described as additional symptoms.

\section{NFOR and OTS as Adjustment Disorders: Maladjustment Syndrome in Athletes}

Literature on NFOR and OTS stresses that they represent "the sum of multiple life stressors, such as physical training, sleep loss, exposure to environmental stresses (e.g., exposure to heat, high 
Table 2 Typical Stressors Found in the Literature That May Cause Adjustment Disorder and Represent Possible Causes in the Development of NFOR or OTS

\begin{tabular}{|c|c|c|}
\hline Characterization & $\begin{array}{l}\text { Occurrence in NFOR } \\
\text { or OTS }\end{array}$ & Comments regarding occurrence in NFOR and OTS literature \\
\hline $\begin{array}{l}\text { Identifiable stressor (DSM-5), signifi- } \\
\text { cant life change, or stressful life event } \\
\text { (ICD-10) }\end{array}$ & $\checkmark$ & \\
\hline single & $\checkmark$ & Stressors are described as stemming from numerous sources, predominantly \\
\hline recurrent & $\checkmark$ & from the sporting environment, including excessive competition and training \\
\hline continuous & $\checkmark$ & loads (exercise workload), monotony of training, and competitive stress \\
\hline multiple (occurring simultaneously) & $\checkmark$ & $\begin{array}{l}\text { etc.), in combination with other stressors (Jones \& Tenenbaum, 2009; } \\
\text { Meeusen et al., 2013; Richardson et al., 2008). }\end{array}$ \\
\hline $\begin{array}{l}\text { Affected integrity of an individual's } \\
\text { social network (ICD-10), such as ... }\end{array}$ & $\checkmark$ & \\
\hline $\begin{array}{l}\text { separation experiences (divorce or } \\
\text { breakup of a relationship) }\end{array}$ & $\checkmark$ & $\begin{array}{l}\text { Quality of relationships with teammates, coaches, and support staff and } \\
\text { parental expectations are named (Jones \& Tenenbaum, 2009). Social envir- }\end{array}$ \\
\hline loss of a loved one & & \\
\hline financial problems & & VanHeest, 2002; Meeusen et al., 2013; Richardson et al., 2008). Illness (most \\
\hline $\begin{array}{l}\text { continuous painful illness } \\
\text { illness that progressively worsens }\end{array}$ & $\checkmark$ & $\begin{array}{l}\text { commonly upper respiratory tract infections; Meeusen et al., 2013; Richardson } \\
\text { et al., 2008). }\end{array}$ \\
\hline \multicolumn{3}{|l|}{ Major developmental transitions such as } \\
\hline$\cdots$ & & \\
\hline marriage & $\checkmark$ & \multirow{6}{*}{$\begin{array}{l}\text { Transitions in the sport, such as new training environments, new levels } \\
\text { (professional), major life events and previous life experiences (sport and } \\
\text { nonsport) such as selection trials, change of marital status, parenthood, change } \\
\text { of residence, etc. (Jones \& Tenenbaum, 2009; Meeusen et al., 2013; } \\
\text { Richardson et al., 2008). }\end{array}$} \\
\hline parenthood & $\checkmark$ & \\
\hline relocating & $\checkmark$ & \\
\hline going to school & $\checkmark$ & \\
\hline leaving home & $\checkmark$ & \\
\hline retirement & $\checkmark$ & \\
\hline Situational crises such as . . . & $\checkmark$ & \multirow{6}{*}{$\begin{array}{l}\text { Severe injury, poor performance, financial problems, failure in study com- } \\
\text { mitments, or other life crises (Richardson et al., 2008). }\end{array}$} \\
\hline losing a job & & \\
\hline failure to pass major exams & $\checkmark$ & \\
\hline failure to succeed in a career & $\checkmark$ & \\
\hline $\begin{array}{l}\text { failure to achieve a cherished personal } \\
\text { goal }\end{array}$ & $\checkmark$ & \\
\hline serious physical illness & & \\
\hline $\begin{array}{l}\text { Micro-stresses: little daily stressors that } \\
\text { accumulate over time }\end{array}$ & $\checkmark$ & $\begin{array}{l}\text { Daily hassles, inadequate nutrition, sleep loss, training monotony, exposure to } \\
\text { environmental stresses (e.g., exposure to heat, high humidity, cold, or high } \\
\text { altitude), occupational pressures, or school- or work-related demands } \\
\text { (Armstrong \& VanHeest, 2002; Jones \& Tenenbaum, 2009; Meeusen et al., } \\
\text { 2013; Richardson et al., 2008). }\end{array}$ \\
\hline
\end{tabular}

Note. $\mathrm{NFOR}=$ nonfunctional overreaching; OTS = overtraining syndrome. Stressors of adjustment disorder based on DSM-5 and ICD-10 criteria.

humidity, cold, high altitude), occupational pressures, change of residence and interpersonal difficulties" (Meeusen et al., 2013, p. 5). This bears a striking resemblance to the etiology of adjustment disorder. Table 2 gives an overview of stressors that may cause adjustment disorder, and these correspond with those responsible for the development of NFOR and OTS. Unsurprisingly, the symptoms and consequences of NFOR, OTS, and adjustment disorder are also almost identical (see Table 1). In addition, many individuals with adjustment disorder recover without treatment, because people have resources to cope with life stressors and adjustment-disorder symptoms (Zelviene \& Kazlauskas, 2018). The same can be said about NFOR and OTS. According to Jones and Tenenbaum (2009), an athlete's overtrained state is more perceptible if it is considered an adjustment disorder. Viewing NFOR and OTS as specific forms of maladjustment widens the number of stressors that can be considered beyond excessive training load, while the key symptom remains prolonged underperformance. This facilitates the holistic treatment of the athletes, viewing them as whole individuals rather than only as athletes.

In this view, NFOR and OTS are regarded as more or less normal reactions (with clinically relevant symptoms) to a series of significant stressors. The expression maladjustment syndrome in athletes takes into account the athletic context, multifactorial etiology, and range of contributing stressors without classifying it as a clinical disorder. While NFOR and OTS, viewed as maladjustment syndromes in athletes, are generally caused by exposure to physiological stress, it is important to recognize that they develop in combination with other stressors, which are often trivialized or neglected by a sport-centered personality. It is also important to recognize that for competitive athletes with an identity 
narrowly focused on their athletic role, experiences of prolonged underperformance and failure to meet important competitive goals may be threatening to self-esteem (Gustafsson, Martinent, IsoardGautheur, Hassmén, \& Guillet-Descas, 2018). Such experiences may be seen as personal failures and may represent a loss of mental power, which an individual may wish to hide and of which he or she may be ashamed (Schwenk, 2000).

An important additional factor in an athletic context is that almost all athletes have found that an increase in training intensity and training volume (training harder and more often) leads to a better, more stable performance. Moreover, most athletes have had experiences of functional overreaching, in which after a period of heavy training load with signs of exhaustion and extreme fatigue, their performance returned to normal or supercompensated. Therefore, and because sport culture promotes being mentally tough and pushing past one's limits, athletes may be tempted to neglect the signs of NFOR and OTS. This aspect of sport culture is characterized by sayings like No pain, no gain and Go hard or go home. These dictums are counterproductive when taken out of context. In this kind of sport culture, athletes may try to hide their performance inadequacy, and it may push them to train excessively to protect their self-esteem (Gustafsson et al., 2018).

\section{Context and Consultancy Philosophy}

I have a master's degree in sport science and a master's degree in general psychology, and I am a sport psychologist certified by the National Federation of Psychologists. In addition, I completed a 1-year research and further education scholarship to investigate the relationship between mood and performance in the context of overtraining phenomena. I have authored research papers in this area (e.g., Birrer et al., 2013). At the time of this case study, I was working in a multidisciplinary sport and service center for elite athletes. In this position, I was conducting research, teaching, and practicing counseling in collaboration with sport scientists and physicians. This environment provided me the opportunity to develop and use advanced scientific knowledge and research from several academic disciplines to render applied services for the specific wants and needs of elite athletes and coaches. However, publication pressure at this institution is low, so various research findings have not been published.

My training in psychology was based primarily on a cognitivebehavioral perspective, so my first steps in designing an intervention were characterized by the application of psychological-skills training tools and cognitive-behavioral therapy (CBT) methods. After several years of working as an applied sport psychologist, I have found CBT methods limited, because I have experienced that sometimes athletes have many problems trying to control their thoughts and emotions. To address this problem, I started to use third-wave CBTs, which integrate mindfulness as a core concept (Hayes, 2004). I have found mindfulness and acceptance-based methods to provide a better understanding of psychological processes, and they seem to foster optimal human functioning. Although third-wave therapies build on behavioral and cognitivebehavioral methods, they suggest that functional behavior change is fostered not necessarily by a change in the content of thoughts and emotions but by a change in an individual's relationship to this content (Hayes, 2004). In addition, both classic CBT and third-wave CBT stress the importance of learning processes in the development and maintenance of functional behavior. Another main feature of third-wave therapies is the assumption that all psychological processes have a function (i.e., a purpose) or had a function during a person's ontogenetic development. In this sense, an individual is regarded as the expert on his or her own life.

To involve athletes in the therapeutic process, third-wave therapies often involve the use of psychoeducation to teach them, for example, why people experience certain thoughts and emotions. This component is essential in softening and changing dysfunctional behaviors and attitudes. In this way, athletes become actively involved in the counseling process, making it an "equal" exchange with the consultant. Coming to understand one's own behavior, which is often experienced as dysfunctional, as a normal, easily explainable psychological process often has a relieving effect by reducing shame and negative feelings experienced by athletes. It also strengthens the interaction and mutual relationship between athlete and psychologist.

\section{Rowing Over the Edge: Greg's Case}

The following case description originates from routine applied services in my consulting practice. The ethical guidelines of the European Federation of Psychologists' Associations were followed. In addition, the ethical guidelines of the National Psychological Association state that members may use information subject to confidentiality anonymously for didactic, statistical, research, or publication purposes. Information is considered anonymous if conclusions about specific clients cannot be drawn or can only be drawn with disproportionate effort. Accordingly, the data have been anonymized so that no unambiguous conclusions can be drawn about the athlete's identity. Because gender is irrelevant in this context, even the athlete's gender may not be accurately reported. As part of another research project, an insight interview was conducted approximately half a year after the athlete's return to competition as part of a series of interviews with formerly diagnosed and psychologically treated NFOR or OTS athletes. The athletes agreed by written consent that the information can be used for didactic, research, and publication purposes.

The consultancy was initiated by another sport psychologist who asked if I would be willing to take over the case of an overtrained athlete. Because I had experience investigating and treating overtraining phenomena as a scientist and practitioner, I agreed. At the beginning of the intervention, the athlete, let us call him Greg, was 21 years old and a member of the under- 23 national team. He had successfully competed at previous junior European and world championships. At the beginning of the consultation during Calendar Week 26, he had cancelled the respective competitive season and was in medical treatment.

\section{Intake and Needs Overview}

Greg was referred by a colleague with whom he had already had two intervention sessions. Greg expressed the wish to work with a sport psychologist who had experience treating athletes with overtraining problems. Issues that had been discussed in the first intervention session with my colleague, which took place during Calendar Week 23, were mental toughness, overtraining, and disturbing factors stemming from the national-team environment and the suicide of a rowing friend. Greg stated in this session that the overtraining had almost been successfully overcome. After this first session with the colleague, Greg had a relapse. After a devastating performance test and the following consultation with a physician, Greg decided to stop training and competing (Week 24). In Week 25, Greg had the second intervention with my colleague, in which he expressed his desire to be referred to a more experienced sport psychologist. 
In Week 26, weekly mood-state and stress-recovery monitoring started. In Week 27, my first intervention session with Greg took place, in which Greg expressed his need to analyze his overtraining and to find a way out of his crisis.

\section{Case Formulation}

Situational and Personal Characteristics. The following are the situational and personal characteristics of Greg's case highlighted from the perspective of NFOR and OTS as special forms of adjustment disorder:

- Significant increase in weekly training volume: $M=16.6 \mathrm{hr}$ (8.75-22.75 hr)

- Suicide of a close friend

- Change from the open category to lightweight rowing (individual weight limit $72.5 \mathrm{~kg}$, team mean limit $70 \mathrm{~kg}$ )

- Change of discipline (scull to one oar)

- Breakup with girlfriend

- End-of-term school examinations

- Change of training environment (from club level to the A-National team)

- Disagreement with national coach concerning the required weight limits

- Perfectionistic personality with very high personal standards

The situational characteristics demonstrate that multiple stressors (see Table 2) were simultaneously present in Greg's life. Some of the stressors were single events, and some of them were recurrent and continuous.

Etiology. Greg's perspective concerning the evolution of his maladjustment was gleaned from notes in his personal training log. To better illustrate Greg's thoughts and feelings, the description is enriched with quotes from that log. Some of the additional information and quotes are from the intervention sessions, as well as from the aforementioned in-depth interview. This analysis begins with data from Calendar Week 13 of the previously referenced year and aims to provide a good overview of the situation and sport culture in which Greg was trapped.

Calendar Week 13: Personal quote: "The national coach set my weight lower than he had promised me a week before the selection races for the $\mathrm{u}-23$ squad. I had to lose 2 kilograms by sweating on that day, but I still managed to win the qualifiers afterward. After the competition, I told him about my weight problems and told him that my doctor and my nutritionist had discouraged me from losing any more. Nevertheless, this did not interest him very much. He insisted that I have to lose more weight. Therefore, I've done what I was asked for."

Week 14: Best performance test ever, personal record in power test, total loss of $7 \mathrm{~kg}$ weight..

Week 15: Light cough.

Week 16: Worsening of cough, weight-reduction procedure, enhanced training intensity. Reduction of training volume. Personal quote: "And thus when I noticed the physical problems I thought I would have to prove me something or so and go on with training. I didn't take that seriously at all and thought, yes, I had a bit of a cough and yes, that will be gone after a few weeks."

Week 17: Worsening of cough, further weight-reduction procedure, enhanced training intensity for competition preparation, weight on race day $2 \mathrm{~h}$ before competition $70.6 \mathrm{~kg}$. First signs of asthma. Further reduction of training volume. Personal quote: "Although I had been suffering from a cough for 2 weeks, I drove two races on the first day of the regatta. Two hours before the race, I had to weigh 70.8 kilograms, which I managed to do by a narrow margin. After the first race with the severe breathing problems, the $\mathrm{u}-23$ team manager advised me to participate in the second race, as well. In the evening, I was very disappointed because I was not honest with myself by rowing the races at all. In addition, I also felt exploited and betrayed."

Week 18: Severe cough, performance inability, asthma. Consultation with a pneumologist with no clinically relevant findings and no appropriate therapeutic treatment options. Further reduction of training volume.

Week 19: Cough slightly better, asthma. Reduced training volume.

Week 20: Light cough, asthma, increase in training volume. Personal quote: "This week, I just had the feeling that despite coughing and a bad mood, I should train again."

Week 21: Worsening of cough, performance inability, and asthma. Renewed reduction of training volume.

Week 22: Stagnation of cough, fatigue, feeling powerless, performance inability, and asthma. Reduction in training volume.

Week 23: First intervention with a sport psychologist. Worst performance test ever (nearly $100 \mathrm{~W}$ less than in Week 14), cough when exercising with heart rate $>120$, increased heart rate and lactate concentration at all test intensities. Feeling powerless, performance inability, asthma.

Week 24: Cough, low sleep quality, fatigue, feeling powerless, performance inability, headache, diarrhea, indisposition, asthma. Consultation with the general physician of the rowing federation. Significant dehydration was diagnosed. Decision to stop training and competing.

Week 25: Cough, low sleep quality, fatigue, feeling powerless, performance inability, headache, diarrhea, indisposition, asthma. Second intervention with a sport psychologist, in which Greg expressed his wish to consult with a psychologist experienced in working with overtrained athletes.

Week 26: Cough, low sleep quality, fatigue, feeling powerless, performance inability, headache, diarrhea, indisposition, asthma. Beginning of mood and stress-recovery monitoring.

Week 27: Same symptomatology. Beginning of counseling.

\section{Holistic Treatment of NFOR/OTS: Treating the Person, Not the Athlete}

Greg was referred to treatment with a diagnosis of NFOR or OTS by a physician and another sport psychologist. Athletes with early signs of NFOR and OTS typically exhibit a specific pattern of mood disturbances, so as suggested by Meeusen et al. (2013), we arranged weekly mood and stress-recovery monitoring. The results of 10 weeks of mood monitoring are presented in Figure 2. Mood monitoring was conducted using Abele-Brehm and Brehm's (1986) Befindlichkeitsskalen, a mood questionnaire similar to the Profile of Mood States (McNair, Droppleman, \& Lorr, 1992). In contrast to the Profile of Mood States-which has one positively connoted scale and five negatively connoted scales-the 


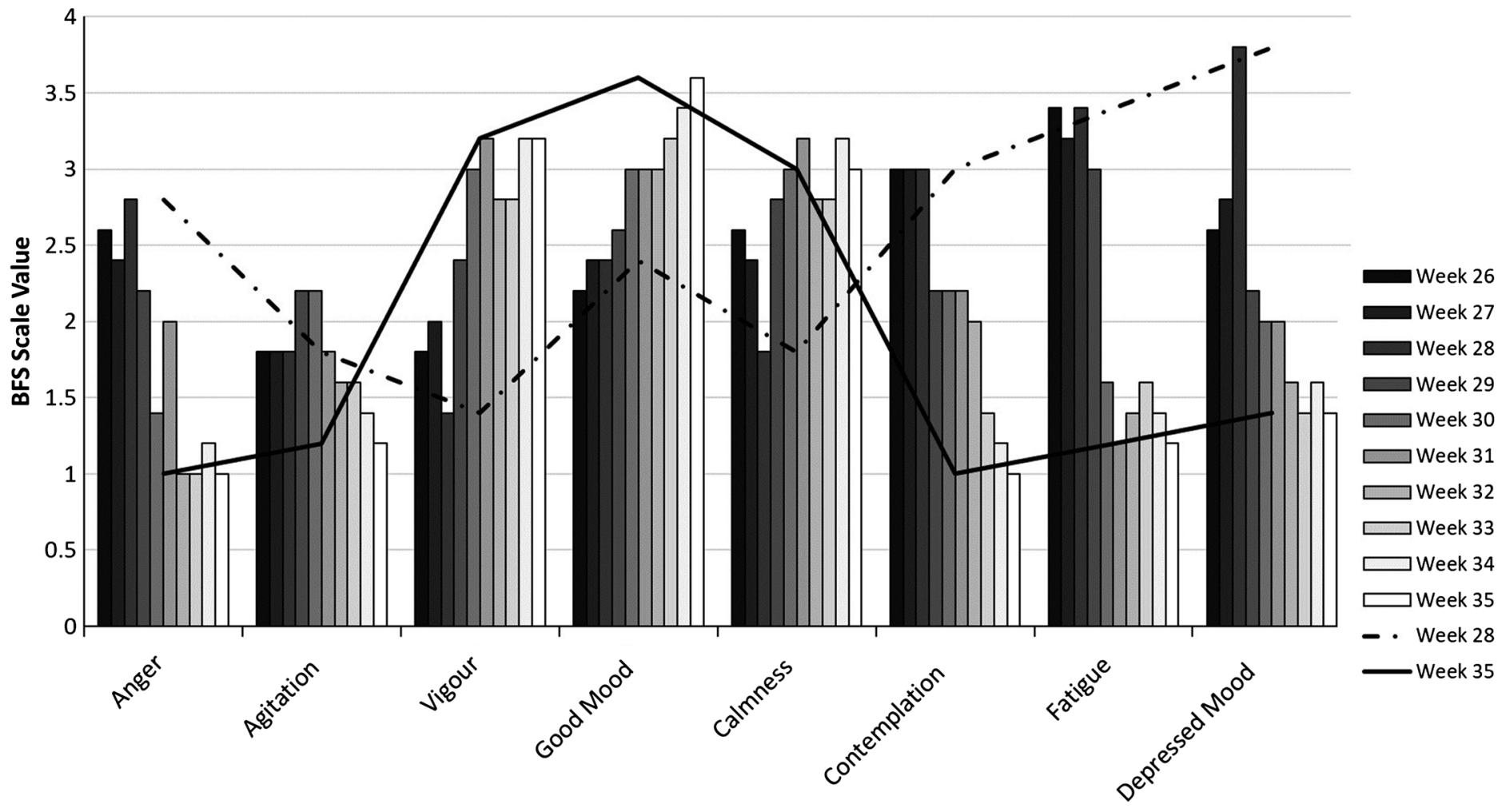

Figure 2 - Magnitude of changes in mood states on the Befindlichkeitsskalen Scale during the 10-week monitoring period (raw values). Lines represent a typical iceberg profile of nonfunctional overreaching (Week 28) and a stress-recovery balance profile (Week 35).

Befindlichkeitsskalen contains four positively and four negatively connoted scales. This allows for a more balanced view of the individual's mood. Mood data revealed that the first weeks of the intervention were characterized by high levels of anger, fatigue, depressed mood, and contemplation; moderately high agitation; moderately low vigor; and low good mood and calmness. This mood combination is symptomatic of NFOR/OTS (Meeusen et al., 2013) and adjustment disorder (American Psychiatric Association, 2013). In addition to the cardinal symptom of significant performance inability and upper respiratory illness, Greg presented a complex combination of single (e.g., suicide of a rowing friend), recurrent (e.g., conflicts with his coaches), and continuous (e.g., significant dietary restriction) stressors stemming from his sport, private life, and educational situation.

Overall, Greg showed several clinically significant symptoms of poor adjustment toward environmental stressors, which can be considered atypical of what would normally be expected given his performance level and training history. His level of distress and impairment reflected a measurable degree of breakdown in biological, psychological, and social functioning. Furthermore, he showed a high level of contemplation reflected in mood monitoring during the first 4 treatment weeks (together with the other mood characteristics; see Figure 2). This suggested preoccupation with the stressor and/or its consequences, including recurrent and distressing thoughts about performance inability and constant rumination about its causes and implications, which are new diagnostic features in the recent ICD-11 definition (World Health Organization, 2018). Furthermore, in the first few intervention sessions, Greg expressed anger toward his coaches and the federation regarding the weight-reduction procedure and other issues.
In summary, a diagnosis of both adjustment disorder and NFOR/OTS or maladjustment syndrome in athletes appeared justified. The following quote offers insight into Greg's state: "When I noticed that I had to break off the season, everything became a little meaningless. I didn't care if the sun was shining or not. My energy tank was empty and remained so for about 2 months. Only through the intervention did my mood slowly improve."

Given that the biopsychosocial nature of maladjustment is characteristic of NFOR and OTS, an appropriate treatment should address all three areas. In addition, Casey and Bailey (2011) reported that "brief therapies are regarded as being the most appropriate, with the exception that, when stressors are ongoing, prolonged supportive measures may be necessary" (p. 15). Reported state-of-the-art treatments for adjustment disorder span a range of supportive psychoeducational, cognitive. and psychodynamic approaches. Casey and Bailey also suggested the following treatments: relaxation techniques to reduce anxiety and techniques to verbalize fears and emotions, explore the meaning the stressor has for the individual, and find alternative responses to stressors when self-destructive behavior is involved. In addition, mindfulness and/or acceptance-based interventions have been shown to be effective (Forman et al., 2012; Srivastava, Talukdar, \& Lahan, 2011; Sundquist et al., 2015) and have been recommended for the treatment of NFOR and OTS by Birrer et al. (2013).

\section{Intervention}

Effective treatment requires an understanding of the interplay between all stressors and the individual's coping resources, so the first intervention session was used, in part, to evaluate Greg's 
personal context. Data concerning his stressors have already been presented. Greg's family was identified as an important personal resource. In the course of identifying Greg's sources of self-esteem, he realized that his only interests were related to rowing. Earlier in his life, he had played an instrument and engaged in other activities besides school and rowing. Due to the great importance rowing had in Greg's life, his performance inability was an extreme threat to his self-esteem. A sport-centered, one-dimensional athletic identity might push athletes to train excessively (Gustafsson et al., 2018), even when they are already fatigued and exhausted. In addition, in this first session Greg discussed what he liked to read and what music was on his play list. He mentioned that he had been reading Nietzsche, and most of the titles in Greg's music play list of this period were in a minor key, which led to the assumption that most activities in which Greg engaged after ceasing training and competition had placed him in a sort of mood-maintenance mode (see mood-behavior model; Gendolla, 2000).

Furthermore, in this first phase of intervention, Greg disclosed many negative emotions. Especially when speaking about his performance inability and situational and personal factors, mood disturbances were dominant, and Greg was tearful and cried several times. Consequently, Greg's task was to engage consciously in a mood-repair mode in which he would deliberately choose activities, music, and readings with a mood-enhancing impact. In addition, he was encouraged to explore activities outside the sport that he perceives as fun and fulfilling.

In accordance with the ECSS and ACSM's (Meeusen et al., 2013) recommendations to treat NFOR and OTS with rest, Greg's physician forbade him from participating in any training activities. Nevertheless, he had a strong desire to be physically active. In collaboration with the physician, it was decided that Greg would be allowed to gradually build in some physical activity. The rule of thumb was that the activity could not be regarded as training but as leisure (e.g., he was allowed to stroll in nature or engage in movement on a whim). Experience in the treatment of overtrained athletes has suggested that it is crucial to reduce not only training volume but also, and more important, training intensity (Meeusen et al., 2013).

Intervention planning was based on my intervention philosophy and training as a psychologist. The intervention comprised a mixture of elements of CBT, psychological-skills training, mindfulness, and acceptance-based interventions (acceptance and commitment therapy, ACT; Hayes, 2004), and the application of harmonious/obsessive passion constructs (Vallerand et al., 2003). To address Greg's history of stressors, much time was invested in sorting out his personal situation with the help of a developmental perspective on the transitions faced by athletes based on Wylleman and Lavallee's (2004) life-span model. Emphasis was placed on Greg's life-balance and life-span management, because transitions always hold the potential for crisis. Values are considered important determinants of human behavior (Hayes, 2004), so a key role in this process was the clarification of Greg's personal values, which is one of the six ACT core processes.

Many methods can be used for value clarification. In this case, the Life Values Inventory (Crace \& Brown, 1996) was used. The results formed the basis for setting goals for the near future (that is, translating values into targeted action; cf. the committed action process of ACT, Hayes, 2004). In this process, Greg realized conflicts between certain values in his life (e.g., conflicts between the fulfillment of health and activity, privacy, and independence on one side and achievement on the other side entailed an intrapersonal conflict leading to feelings of ambivalence). The conscious recognition of conflicts is an important step in increasing self-concordance. High self-concordance is associated with more efficient goal pursuit and goal achievement (Koestner, Lekes, Powers, \& Chicoine, 2002), because unresolved issues get in the way of value fulfillment and enhance psychological inconsistency. The aim is to make a person aware of his or her affective network, link it to everyday life, and harmonize it with superordinate association patterns (e.g., self, reality, or cultural values). The aim of these considerations is to recognize conflicts as far as possible before the formation of intentions. This should facilitate an increase in self-concordance (Koestner et al., 2002).

Enhancement of personal meaning was used to help Greg develop his one-dimensional athletic identity into a multidimensional personality (see self as concept process in ACT; Hayes, 2004). This diversifies the sources on which one's self-esteem is built. In Grawe's (2007) consistency theory, the need for self-esteem enhancement or self-esteem protection is one of four basic psychological needs. Grawe's theory states that the human system strives to avoid inconsistency. Inconsistency leads to psychological dysfunction and maladaptive behavior in the short term and mental disorder in the long term. Value clarification enhances congruency and psychological functioning.

Methods to consciously be in the present moment (mindfulness exercises) and psychological-acceptance strategies (e.g., viewing thoughts and emotions as acceptable as they are, with no need to alter or reduce them) were part of Greg's intervention program from the beginning. These two core ACT and mindfulness processes enhanced Greg's awareness of training intensity and recovery aspects (including subjective feelings affecting pain, fatigue, and recovery), which was also an important goal of the intervention, especially when Greg gradually returned to training. This process was accompanied by psychoeducation on the function of mood, which was facilitated by evaluations using the results of the aforementioned mood monitoring.

Greg was also taught autogenic training to facilitate the reestablishment of his emotional balance and enhance his recovery capacity. Both autogenic training sessions and mindfulness meditations were recorded on tape, and Greg was able to use the records at home. The autogenic training session lasted $22 \mathrm{~min}$ at the beginning, which was gradually reduced to a length of $10 \mathrm{~min}$. To check the physiological effects of the autogenic training, autogenic training sessions were monitored using biofeedback equipment at the beginning of counseling and 10 weeks later. According to his training log, Greg executed 31 autogenic training sessions in a 10-week period. Because most of the mindfulness meditations were informal in nature (e.g., mindful rowing), Greg did not log these sessions systematically. In addition, because people are normally fused with their thoughts, emotions, and bodily sensations (Hayes, 2004)—which is especially crucial for dysfunctional thinking and dysfunctional attempts to control unpleasant feelings-methods of acceptance and cognitive defusion were adopted to increase Greg's ability to step back psychologically from his distressing internal experiences (thoughts, emotions, and bodily sensations) and view them as functional processes rather than absolute truths (Hayes, 2004).

Resumption of training was individualized on the basis of previously agreed-on physical and psychological signs and symptoms (supported by the mood monitoring), with clear criteria of progression to the next training level, stagnation on the same level, or returning to the previous level. Communication with the coach was facilitated by selecting a new personal coach who was 
thoroughly familiar with Greg's maladjustment history and allowed him to discuss his physical, mental, and emotional concerns. In this phase, it was important to help Greg deal with overmotivation in training situations. It seems that for athletes returning to training after NFOR or OTS, it is difficult to train in the planned intensities, especially when training in a group setting. Not being able to train with the same intensities as one's training partners is self-esteem threatening (Armstrong \& VanHeest, 2002). Consequently, athletes in an NFOR or OTS state or in recovery from such a state are always in danger of training at higher intensities than planned because their already weakened self-esteem will suffer even more when they must fall behind their training partners to maintain the intended training intensity. To tackle this challenge, methods of committed action were used (Hayes, 2004). Accordingly, implementation intentions (Gollwitzer \& Sheeran, 2006) were adopted with success. In addition, mindful rowing was established as a form of informal mindfulness training, which also allowed Greg to exercise this core psychological-change process in the environment in which the behavioral change was needed.

Normally, athletes become engaged with their sport because they feel pleasure while doing it, and their basic psychological needs (Grawe, 2007) are nourished by the activity. Nevertheless, in controlling, overdemanding, and rejecting environments, the internalization of behavioral regulations might be characterized by intra- and interpersonal pressure and might become "obsessive" (Vallerand et al., 2003). People with obsessive passions pursue the activity eagerly and compulsively because of the reassurance and ego-affirming moments it provides. Athletes with an obsessive passion for their sport feel compelled to engage in their activity, which enhances their risk of engaging in dysfunctional behavior connected with their sport. It is reported that people with a more obsessive passion experience more conflicts with other life activities, tend to engage in their activity even when the circumstances suggest that engagement is harmful, and have more ruminative thoughts about the activity and lower levels of positive affect (Mageau et al., 2009). Greg, like many NFOR and OTS athletes, also showed many signs of obsessive passion. Consequently, we worked on the rediscovery of Greg's intrinsic motivation, which was also connected to his selection of a new personal coach. Together with the identification of personal values, we discussed the initial reasons Greg was playing his sport. Two key ideas were used in this process: "Allow that rowing is fun!" and "Consider rowing a game."

In the stage during which Greg was able to start training in the boat again-which was approximately 13 weeks after he was forced to end his season-time was invested to work on strategies to enhance training quality, as well as his self-awareness regarding signs of stress and recovery. Overall, the interventions were targeted to allow Greg to be a whole person who is also an athlete and to be able to live a rich and value-based life.

\section{Evaluation of the Intervention and Its Outcomes}

After the treatment, Greg was able to pursue his career by competing at major international events such as world championships and the Olympic Games. Approximately 1 year after he had to end his season because of NFOR/OTS, Greg was rowing his first competition after the OTS. In that year, he received $14 \mathrm{hr}$ of counseling spread over 10 sessions. Nevertheless, the return to training and competition took longer than he expected. As can be seen in Figure 2, Greg's mood states progressively returned to normal over the 10 weeks of monitoring. In addition, some mood scales appear to have changed in a stepwise fashion (e.g., contemplation).
During an interview half a year after his return to competition, Greg talked about the significant factors in the counseling process:

It was important for me that I had been challenged by the sport psychologist with difficult and personal questions, which forced me to more and intense reflection. ... Another decisive factor was certainly that I had to deal with my values and visions of the future and could no longer think only in the context of rowing. This certainly helped me not to see everything in the context of sport anymore, to think beyond the present state and not to regard it as my permanent lot. . . . Afterward through the relaxation and mindfulness techniques, I learned to promote recovery.

Greg offered the following advice to other athletes: "Sometimes it takes more courage to skip a workout than to just stubbornly go through the training plan."

\section{Reflections, Lessons Learned, and Implications}

Viewing Greg's OTS as a training maladjustment in an athletesimilar to a subclinical or clinical form of adjustment disorderwidened the search for symptomatology and causes beyond the phenomenon of too much training. Supporting evidence for maladjustment includes environmental conditions; a "no pain, no gain" or "no excuses" culture; the presence of burdening psychosocial factors; nutritional issues and viral diseases, especially upper respiratory tract infections; a significant increase in training load within the last 6 months; and personality characteristics including athletic identity, perfectionism, or obsessive sport-related passion. These were all present in Greg's case.

In treatment, the goal was to enhance Greg's awareness of his basic biopsychosocial processes, facilitate his acceptance of unpleasant physical and emotional sensations, tackle his overreaching athlete identity, and attend to his predominant performance-related sources of self-esteem. In this process, Greg's values served as a kind of guideline on which to focus his behavioral goals (something one wants to do) as opposed to his outcome goals (something one wants to have). Values include global internal representations of what is considered personally right and important in life (Hayes, 2004), so value clarification helped Greg learn to satisfy his basic psychological needs. Greg felt challenged by personal questions and was forced to engage in more and intense reflection, which he perceived as helpful. In addition, mindfulness and acceptance-based interventions seemed well suited to achieving the mentioned objectives.

In terms of a reflection on the intervention, my background as a psychologist who researches and treats overtraining using mood monitoring, has personal training with classic CBT, and uses mindfulness and acceptance-based interventions influenced the choice of intervention and overall decision making. It also helped me gain the athlete's trust and strengthen treatment compliance. In addition, although Greg found it helpful that I challenged him with personal and difficult questions to engage him in more and intense reflection and to recognize, legitimize, and work through difficult issues, this was a potential pitfall and endangered the working alliance. For example, it was a challenge to empathize with Greg and take his personal dreams and goals seriously while also challenging his athletic identity by relativizing the value of an athletic career, including the value of physical performance and athletic success. So, to engage Greg in a joint exploration of 
obstacles without straining the working alliance, I tried to deliberately nurture Greg's basic psychological needs (Grawe, 2007) during counseling. When Greg expressed a strong need to exercise, I supported him with this wish against the initial prescription of his physician. Although I was convinced that it was important for Greg to integrate a small amount of physical activity into his weekly routines, I could not be sure this was the right decision. Weekly mood monitoring was helpful in estimating Greg's physical and psychological development and provided some confirmation that integrating physical activity was the right decision.

Exploring new sources to nourish Greg's basic psychological need for self-esteem enhancement was also significant, as it was striking for both Greg and me that he had no sources other than his athletic career at that time. In this process, I learned that NFOR/ OTS represents a significant personal crisis. In this respect, and given Greg's tearfulness during the first few intervention sessions, sport psychologists are well advised to prepare for this. A selfcompassionate stance might be helpful in coping with these situations.

\section{Conclusion}

Greg's case demonstrated that an "overtrained state" can be caused by a combination of any simultaneously occurring stressors of any severity and that it must be understood in terms of these combined influences in the respective sport culture. Results of mood monitoring suggested that moods are an important indicator of a person's overall condition and should always be taken seriously as behavioral indicators. This was also reflected by Greg's neglect of his decreasing mood states and other warning signs during the course of his maladjustment. Performance and mood disturbances are key symptoms of training maladaptation, so monitoring an athlete's mood and stress-recovery state appears to be very useful in the support of elite athletes. Athletes, as well as coaches, psychologists, and psychology trainees, should be taught that mood states provide significant information.

To treat NFOR/OTS effectively, practitioners must shift their focus from training and recovery schedules to a comprehensive understanding of the athlete's holistic biopsychosocial stressresponse process (Jones \& Tenenbaum, 2009). This widens treatment options to include psychological and social strategies. The person as a whole - and not just the athlete-will be treated with respect to his or her personal history, values, and the role of sport in his or her present and future life. Overall, Greg benefitted from psychological and behavioral flexibility by developing a constructive response to stress (Hayes, 2004), which is supported by recent research (Chang, Wu, Kuo, \& Chen, 2018) suggesting that psychological flexibility prevents emotional exhaustion in subjects with high levels of athletic identity.

\section{References}

Abele-Brehm, A., \& Brehm, A. (1986). Zur Konzeptualisierung und Messung von Befindlichkeit. Die Entwicklung der "Befindlichkeitsskalen" (BFS) [The development of the "mood scales" (MS)]. Diagnostica, 32, 209-228.

American Psychiatric Association. (2013). Diagnostic and statistical manual of mental disorders (DSM-5). Arlington, TX: American Psychiatric Publishing.

Armstrong, L. E., \& VanHeest, J. L. (2002). The unknown mechanism of the overtraining syndrome-Clues from depression and psychoneuroimmunology. Sports Medicine, 32(3), 185-209. PubMed ID: 11839081 doi:10.2165/00007256-200232030-00003
Birrer, D., Lienhard, D., Williams, C. A., Röthlin, P., \& Morgan, G. (2013). Prevalence of non-functional overreaching and the overtraining syndrome in Swiss elite athletes. Schweizerische Zeitschrift für Sportmedizin \& Sporttraumatologie, 61(4), 23-29.

Bosquet, L., Montpetit, J., Arvisais, D., \& Mujika, I. (2007). Effects of tapering on performance: A meta-analysis. Medicine and Science in Sports and Exercise, 39(8), 1358-1365. PubMed ID: 17762369 doi:10.1249/mss.0b013e31806010e0

Casey, P., \& Bailey, S. (2011). Adjustment disorders: The state of the art. World Psychiatry, 10(1), 11-18. PubMed ID: 21379346 doi:10.1002/ j.2051-5545.2011.tb00003.x

Chang, W. H., Wu, C.-H., Kuo, C.-C., \& Chen, L. H. (2018). The role of athletic identity in the development of athlete burnout: The moderating role of psychological flexibility. Psychology of Sport and Exercise, 39, 45-51. doi:10.1016/j.psychsport.2018. 07.014

Crace, R. K., \& Brown, D. (1996). Life values inventory. Minneapolis, MN: National Computer Systems.

Forman, E. M., Chapman, J. E., Herbert, J. D., Goetter, E. M., Yuen, E. K., \& Moitra, E. (2012). Using session-by-session measurement to compare mechanisms of action for acceptance and commitment therapy and cognitive therapy. Behavior Therapy, 43(2), 341-354. PubMed ID: 22440070 doi:10.1016/j.beth.2011.07.004

Gabbett, T. J. (2016). The training-injury prevention paradox: Should athletes be training smarter and harder? British Journal of Sports Medicine, 50(5), 273-280. PubMed ID: 26758673 doi:10.1136/ bjsports-2015-095788

Gendolla, G. H. (2000). On the impact of mood on behavior: An integrative theory and a review. Review of General Psychology, 4(4), 378-408. doi:10.1037/1089-2680.4.4.378

Gollwitzer, P. M., \& Sheeran, P. (2006). Implementation intentions and goal achievement: A meta-analysis of effects and processes. Advances in Experimental Social Psychology, 38, 69-119. doi:10.1016/ S0065-2601(06)38002-1

Grawe, K. (2007). Neuropsychotherapy: How the neurosciences inform effective psychotherapy. Mahwah, NJ: Lawrence Erlbaum.

Gustafsson, H., Martinent, G., Isoard-Gautheur, S., Hassmén, P., \& Guillet-Descas, E. (2018). Performance based self-esteem and athlete-identity in athlete burnout: A person-centered approach. Psychology of Sport and Exercise, 38, 56-60. doi:10.1016/j. psychsport.2018.05.017

Hayes, S. C. (2004). Acceptance and commitment therapy, relational frame theory, and the third wave of behavioral and cognitive therapies. Behavior Therapy, 35(4), 639-665. doi:10.1016/S0005-7894(04) 80013-3

Jones, C. M., \& Tenenbaum, G. (2009). Adjustment disorder: A new way of conceptualizing the overtraining syndrome. International Review of Sport \& Exercise Psychology, 2(2), 181-197. doi:10.1080/ 17509840903110962

Koestner, R., Lekes, N., Powers, T. A., \& Chicoine, E. (2002). Attaining personal goals: Self-concordance plus implementation intentions equals success. Journal of Personality and Social Psychology, 83(1), 231-244. PubMed ID: 12088128 doi:10.1037/0022-3514. 83.1 .231

Kreher, J. B. (2016). Diagnosis and prevention of overtraining syndrome: An opinion on education strategies. Open Access Journal of Sports Medicine, Volume 7, 115-122. PubMed ID: 27660501 doi:10.2147/ OAJSM.S91657

Mageau, G. A., Vallerand, R. J., Charest, J., Salvy, S.-J., Lacaille, N., Bouffard, T., \& Koestner, R. (2009). On the development of harmonious and obsessive passion: The role of autonomy support, activity specialization, and identification with the activity. Journal of 
Personality, 77(3), 601-646. PubMed ID: 20078732 doi:10.1111/j. 1467-6494.2009.00559.x

McNair, D. M., Droppleman, L. F., \& Lorr, M. (1992). Profile of Mood States manual (rev. ed.). San Diego, CA: Educational and Industrial Testing Service.

Meeusen, R., Duclos, M., Foster, C., Fry, A., Gleeson, M., Nieman, D., . . . Urhausen, A. (2013). Prevention, diagnosis and treatment of the overtraining syndrome: Joint consensus statement of the European College of Sport Science (ECSS) and the American College of Sports Medicine (ACSM). European Journal of Sport Science, 13(1), 1-24. doi:10.1080/17461391.2012.730061

Morgan, W.P., Brown, D.R., Raglin, J.S., O’Connor, P.J., \& Ellickson, K.A. (1987). Psychological monitoring of overtraining and staleness. British Journal of Sports Medicine, 21(3), 107-114. PubMed ID: 3676635 doi:10.1136/bjsm.21.3.107

Richardson, S. O., Andersen, M., \& Morris, T. (2008). Overtraining athletes: Personal journeys in sport. Champaign, IL: Human Kinetics.

Schwenk, T. L. (2000). The stigmatisation and denial of mental illness in athletes. British Journal of Sports Medicine, 34(1), 4-5. PubMed ID: 10690441 doi:10.1136/bjsm.34.1.4

Srivastava, M., Talukdar, U., \& Lahan, V. (2011). Meditation for the management of adjustment disorder anxiety and depression. Complementary Therapies in Clinical Practice, 17(4), 241-245. PubMed ID: 21982141 doi:10.1016/j.ctcp.2011.04.007
Sundquist, J., Lilja, Å., Palmér, K., Memon, A. A., Wang, X., Johansson, L. M., \& Sundquist, K. (2015). Mindfulness group therapy in primary care patients with depression, anxiety and stress and adjustment disorders: Randomised controlled trial. British Journal of Psychiatry, 206(2), 128-135. PubMed ID: 25431430 doi:10.1192/bjp.bp.114. 150243

Vallerand, R. J., Blanchard, C., Mageau, G. A., Koestner, R., Ratelle, C., Leonard, M., . . Marsolais, J. (2003). Les passions de l'Ame: On obsessive and harmonious passion. Journal of Personality and Social Psychology, 85(4), 756-767. PubMed ID: 14561128 doi:10.1037/ 0022-3514.85.4.756

World Health Organization. (2016). International statistical classification of diseases and related health problems (ICD-10)-WHO (10th rev., 5th ed., version 2016). Retrieved from https://icd.who.int/browse10/ 2016/en\#/F43

World Health Organization. (2018). ICD-11 for mortality and morbidity statistics (ICD-11 MMS) (11th rev., version 2018). Retrieved from https://icd.who.int/browse11/1-m/en

Wylleman, P., \& Lavallee, D. (2004). A developmental perspective on transitions faced by athletes. In M. Weiss (Ed.), Developmental sport and exercise psychology: A lifespan perspective (pp. 507-527). Morgantown, WV: Fitness Information Technology.

Zelviene, P., \& Kazlauskas, E. (2018). Adjustment disorder: Current perspectives. Neuropsychiatric Disease and Treatment, 14, 375-381. PubMed ID: 29416339 doi:10.2147/NDT.S121072 